\title{
Mecanismos de limitação da energia de raios cósmicos durante sua propagação
}

\author{
Mechanisms for energy limitation of cosmic rays during propagation \\ Yan Bueno Bandeira ${ }^{10}$, Luiz Fernando Mackedanz ${ }^{*}[0$ \\ ${ }^{1}$ Universidade Federal do Rio Grande Instituto de Matemática, Estatística e Física, Rio Grande, RS, Brasil \\ ${ }^{2}$ Universidade Federal do Rio Grande, Programa de Pós-Graduação em Educação em Ciências, Instituto de Matemática, \\ Estatística e Física, Rio Grande, RS, Brasil
}

Recebido em 20 de Maio, 2019. Aceito em 27 de Maio, 2019.

\begin{abstract}
A cada segundo que passa, um número muito grande de partículas de origem cósmica atravessam o planeta. A maior parte delas pouco interage com a matéria e sua passagem não é percebida, exceto pelos pesquisadores da física de partículas. Os raios cósmicos são um tipo destas partículas, de origem extraterrestre, que podem ser estudados com auxílio de detectores. Neste trabalho, apresentamos um estudo sobre as formas como os raios cósmicos interagem durante sua propagação até a Terra, levando a um limite de energia acessível para detectores no solo. Utilizando apenas conhecimentos de Eletromagnetismo e de Relatividade Restrita, buscamos explicar este mecanismo para estudantes que tenham interesse na área, mas que ainda não aprofundaram estudos em física de partículas. Nosso objetivo é apresentar um material didático e acessível para estes interessados.
\end{abstract}

Palavras-chave: Corte GZK, eletromagnetismo, física de partículas.

A very large number of particles of cosmic origin cross the planet every second. Most of them rarely interact with matter, and their passage is not perceived except by researchers of particle physics. Cosmic rays are a type of these particles, of extraterrestrial origin, that can be studied with the help of detectors. In this work, we present a study on the ways in which cosmic rays interact during their propagation to Earth, leading to an accessible energy limit for detectors in the ground. Using only Electromagnetism and Special Relativity knowledge, we seek to explain this mechanism to students who have an interest in the field, but who have not yet studied in particle physics. Our objective is to present a didactic and accessible material for these interested parties.

Keywords: GZK cutoff, electromagnetism, particle physics.

\section{Introdução}

Ao longo da graduação de cursos de Física, o estudo de processos envolvendo altas energias, mesmo quando tratados exclusivamente sob a Teoria Eletromagnética, são deixados para o final do curso, onde espera-se que os estudantes tenham base científica e matemática para entender os processos associados.

Por outro lado, existem muitos processos básicos que são ancorados em leis de conservação que podem ser apresentados mais cedo aos estudantes, sobretudo quando envolvidos em montagens experimentais ou em tratamento de dados experimentais. É nesta linha que entendemos se encontrar a física dos raios cósmicos. Além disso, a utilização de aspectos avançados de pesquisa pode significar trabalhar com conceitos mais próximos dos estudantes, sem se afastar do campo de pesquisa e das últimas inovações científicas [1].

Apesar desta área também ser associada a um entendimento profundo de física nuclear e de partículas, existe uma componente eletromagnética associada aos proces- sos que pode ser explorada anteriormente na formação dos estudantes. Mais do que ser aplicados apenas para interessados em prosseguir na área, estes processos permitem um entendimento da interação eletromagnética da matéria, tanto na propagação dos raios cósmicos como em sua detecção.

Para ilustrar isso, neste artigo tratamos de efeitos de limitação de energia durante a propagação, buscando explicar, apenas com uma base do Eletromagnetismo e da Relatividade Especial, o mecanismo do corte GZK 2.3], que limita a energia dos raios cósmicos detectados na superfície terrestre. Inicialmente, apresentamos um histórico sobre a descoberta dos raios cósmicos, seguindo a lógica internacionalista da realização da ciência. A seguir, nos dedicamos a apresentar brevemente possíveis fontes para estas partículas e, ao final, descrevemos a propagação e os mecanismos de limitação de energia.

Nosso intento, com este artigo, é proporcionar material de consulta, básico, para ingressantes na área e para interessados em compreeender alguns dos processos básicos (já explorados em trabalhos anteriores [4]) da física

*Endereço de correspondência: luismackedanz@furg.br 
de partículas, ainda que não descritos pelas interações fortes.

\section{A descoberta dos raios cósmicos}

Em junho de 1911, o físico italiano Domenico Pacini (1878-1934) introduziu um eletroscópio nas águas do Golfo de Gênova para medir a condutividade residual do ar contido no interior no aparelho e acabou por concluir que os resultados diferentes do esperado baseado nas informações da época, deveriam ser provenientes de um agente extraterrestre [5,6]. Poucos meses depois, o física austríaco Viktor Franz Hess (1883-1964), de forma independente, em experimentos realizados a bordo de balões em altitudes maiores, também caracterizou esta radiação de forma semelhante [7].

O contexto histórico científico que leva Pacini a realizar este experimento remonta aos primórdios da Teoria Eletromagnética. Em 1785, com a apresentação do trabalho pelo físico francês Charles Augustin de Coulomb (1736-1806) de que uma esfera eletrizada, carregada e em ambiente fechado, suspensa por um fio de seda perdia carga de maneira progressiva, o que apontava para a existência de uma condutividade intrínseca do ar. Michael Faraday (1791-1867), em 1835 e William Crookes (1832-1919), em 1879, observaram que a velocidade de descarga era proporcional à pressão do ar, corroborando a ideia da ionização do ar atmosférico.

Em 1896, Henri Becquerel (1852-1908) verificou que sais de urânio emitiam raios que eram capazes de aumentar a condutividade do ar, os quais foram denominados raios ionizantes, pois tinham a capacidade de retirar elétrons dos átomos e de gases, dando origem a íons livres. Logo após da descoberta de Becquerel, Pierre Curie (1859-1906) e Marie Curie (1867-1934) descobriram que outras substâncias eram capazes de emitir esses raios espontaneamente, chamando-as de substâncias radioativas 8]. Com isso, permitiu-se determinar a velocidade de ionização a partir da taxa de descarga de um eletroscópio, usando a radioatividade como padrão de calibragem.

No início do século XX, Charles Thomson Rees Wilson (1869-1959), na escócia, e Hans Geitel (1855-1923) e Julius Elster (1854-1920), na Alemanha, realizaram montagens experimentais com eletroscópios de folhas de ouro, uma versão mais sofisticada e evoluída da esfera de Coulomb, e ambos constataram uma descarga de maneira indefinida e de velocidades variadas, mesmo com o máximo de cuidado para manter isoladas as folhas do eletroscópio [9]. Assim, era possível fazer a medição da taxa de descarga com maior precisão, o que permitia medidas mais acuradas sobre a ionização do ar atmosférico. Assim, em 1902, Ernest Rutherford (1871-1937) e seus colaboradores mostraram que a radiação ionizante era externa ao instrumento; ela era muito penetrante e não provinha da radioatividade das superfícies interiores. Com isto se deu início a busca por tal fonte radioativa que produziria tal emissão, caracterizando seu tipo e sua origem. Uma origem óbvia, para a comunidade cientifica, era de que esta fonte fosse a radioatividade natural da crosta terrestre. Uma prova experimental, porém, era difícil de ser conseguida e, por esse motivo, o assunto seguia aberto a especulações [9].

Numa destas hipóteses, Charles Wilson, em tom profético, sugeriu um possível origem extraterrestre para tal radiação, porém sua montagem experimental, em túneis de grandes profundidades, sob uma camada de rocha sólida, não resultavam em nenhum decréscimo na ionização, o que não suportava a origem extraterrestre desta radiação. Esta hipótese, porém, não é completamente abandonada, uma vez que é retomada em 1907 pelo engenheiro químico Francês Albert Nodon (1862-1934) 8]. Nodon é considerado um dos pioneiros na física da radiação cósmica, realizando estudos precisos das variações de ionização segundo a altitude, assumia que esta radiação penetrante tinha origem exterior a Terra.

Resumindo o que havia sido detectado experimentalmente e seu desenvolvimento teórico quanto à ionização do ar, três fontes eram possíveis: uma radiação extraterrestre, provavelmente proveniente do Sol; a radioatividade da crosta terrestre; e a radioatividade da atmosfera. Isto foi sumarizado por Karl Kurz em 1909 10], que concluiu, a partir de experimentos realizados na parte mais baixa da atmosfera, que a fonte extraterrestre para a radiação era improvável [9]. Neste mesmo ano, o físico Theodor Wulf (1868-1946), inventor de eletroscópio, decidiu investigar a variação da radioatividade com a altura, de forma a testar sua origem. Para isso, levou um de seus aparelhos para a torre Eiffel e realizou medições de ionização do ar tanto no topo da torre (a 300m de altura) quanto na base. Esperava-se que, a partir de $80 \mathrm{~m}$ de altura, a taxa de ionização diminuísse à metade, mas Wulf constatou que a taxa medida era bastante superior ao valor esperado. Para justificar tal fato, ele concluiu que ou haveria fontes de raios $\gamma$ na atmosfera, ou a absorção de radiação $\gamma$ no ar era muito mais fraca do que se pensava [8].

Os resultados obtidos por Wulf foram de grande valor para a ciência, uma vez que forma coletados em diferentes horários do dia e por um número grande de dias. Isso forneceu confiabilidade à informação do efeito da altitude sobre a radiação penetrante. Porém, mesmo com este resultado inesperado, Wulf manteve a conclusão de que a explicação mais provável ainda era a emissão a partir do solo 9 .

\subsection{A contribuição de Pacini}

Esta conclusão começou a ser contestada por Domenico Pacini, a partir de comparações entre taxas de ionização medidas em altitude e embaixo d'água. Seus estudos foram desenvolvidos no período entre 1909 e 1911, principalmente buscando medidas da condutividade do ar, usando um eletroscópio mais sensível 9].Nesta sequência de medições, buscou estudar também a dependência no tipo de meio envolvido na descarga. Seus experimentos 
sob a água não podiam ser explicados pelo modelo da emissao de radioatividade pelo solo, considerando esta como com taxa constante, sendo o pioneiro nesta determinação [11.

Realizando experimentos detalhados sob o Golfo de Gênova, a 300m da costa, a $3 \mathrm{~m}$ de profundidade, e utilizando um eletrômetro disposto em uma caixa de cobre, que possibilitava sua imersão na água a tal profundidade, Pacini comparou as taxas de descargas medidas pelo aparato na superfície e submerso, constatando um decréscimo de $20 \%$ em relação ao valor na superfície. Isto era consistente com a absorção pela água de radiação proveniente de uma fonte externa. Segundo Pacini [5, 6], ao utilizar um coeficiente de absorção para a água igual à 0,034 , e considerando a pouca presença de substâncias radioativas no mar e as próprias condições do experimento, com a caixa de cobre que envolvia o aparato, o resultado leva a conclusão da existência de uma radiação altamente penetrante e não proveniente da superfície terrestre, ou da atmosfera, tendo em mente que a água absorveria tal radiação. Por esse motivo, a radiação medida no experimento de Pacini era uma radiação exterior, extraterrestre 8, 11, 12].

Este resultado de Pacini, porém, apesar de ser relevante para o entendimento da origem da radiação ionizante, ainda não pode ser considerado como definitivo, uma vez que a técnica utilizada não excluía totalmente a origem atmosférica da radiação [8]. Um ano após o trabalho de Pacini, foram obtidos resultados mais precisos sobre a origem da radiação ionizante, a partir de medições a altas altitudes realizando ascensões em balões. Experimentos deste tipo foram realizadas primeiramente pelo físico alemão Albert Gockel (1860-1927), e tinham como motivação a mesma de Wulf: proteger seus equipamentos dos efeitos da radiação do solo. E assim como Wulf observou, Gockel percebeu que por mais densa que fosse a massa de ar durante as ascensões, isso não afetava os resultados com um decréscimo da ionização. Estes resultados, porém, em conjunto com os obtidos por Pacini, apontavam a necessidade de considerar, de forma sistemática, uma fonte diferente da crosta terrestre para explicar os resultados, o que os físicos da época ainda relutavam em aceitar 9 .

\subsection{A contribuição de Hess}

Os resultados obtidos por Wulf foram posteriormente questionados pelo físico inglês Arthur Stewart Eve (18621948), ao estudar os coeficientes de absorção da radioatividade na atmosfera. Segundo Eve, se assumirmos uma distribuição uniforme de $\mathrm{RaC}$ (cloreto de rádio) na superfície e na camada mais externa da Terra, o efeito da radiação decresce para 36\% com uma elevação de 100 $\mathrm{m}$ [9].

Essas observações serviram de motivação para o físico austríaco Viktor Franz Hess (1883-1964), pois o levou a medir o coeficiente de absorção de raios $\gamma$ pelo ar em uma série de experiências em laboratório. Nestas montagens experimentais, este coeficiente variava entre uma fonte intensa de radiação e um detector de ionização e um eletrômetro de Wulf. O resultado desta série de experiências permitiu inferir que a radiação deveria ser absorvida pelo ar a altitudes maiores do que $500 \mathrm{~m}$, supondo a crosta terrestre como a fonte intensa 8.

Tendo em mãos este resultado, Hess começa a fazer experiências em balões entre 1911 à 1912, com várias ascensões, sendo em sua maioria a noite, com apenas uma delas durante o dia, porém sob eclipse total, o que permitiu ao mesmo desconsiderar o Sol como fonte direta de radiação. Em 1912, munido de um eletroscópio, agora associado a um detector de ionização, Hess realizou medições na altura de $5.350 \mathrm{~m}$. Ali constatou que os efeitos ionizantes aumentavam de maneira evidente, e este aumento não era correlacionado a fontes atmosféricas ou terrestres. Como este aumento era proporcional a altura obtida pela balão, ficou claro que essa radiação ionizante tinha realmente fonte extraterrestre [7]. Hess realizou medições em várias altitudes, com cuidado especial para minimizar a radiação de seu próprio dispositivo de medidas, assim como levar as variáveis meteorológicas em consideração.

Ao fazer a análise de seus dados, sua primeira conclusão foi de que para altitudes de aproximadamente $1000 \mathrm{~m}$ haveria uma diminuição na medida de radiação devido ao fato de que os raios $\gamma$ provenientes da superfície terrestre seriam absorvidos pelo ar, assim como previsto pelo mesmo. O que não podia ser explicado era o comportamento contrário para altitudes maiores, conforme aumentava a altitude maior era a radiação encontrada, e essas observações não podiam ser explicadas nem pelas substâncias radioativas presentes nas nuvens ou nos nevoeiros e nem pela variações meteorológicas. Portanto, restava somente uma explicação, haveria uma radiação penetrante que alcançava nossa atmosfera pelo alto e produziria nas camadas atmosféricas, até mesmo nas mais baixas uma quantidade de ionização significativa a ponto de ser observada em recipientes fechados 8 .

\subsection{Polêmicas e o Prêmio Nobel}

Inicialmente essa nova radiação foi denominada de radiação ultrapenetrante, pela sua capacidade de atravessar grandes quantidades de matéria, mas sua denominação mais usual e precisa em relação ao conhecido atualmente deu-se inicio uma década após os experimentos de Pacini e Hess, com o termo radiação cósmica usado pelo físico norte-americano Robert Millinkan (1868-1953). Aliás, Millikan e Hess estiveram envolvidos em uma polêmica quanto à prioridade da descoberta, no final da década de 1920. Utilizando sua reputação como propaganda, os raios cósmicos eram conhecidos como Raios Millikan em muitos países europeus, como a França. Devido às contribuições do francês Nodon aos estudos da radiação cósmica, chegaram a ser denominados Raios Millikan- 
Nodon neste país 8]. Apesar desta denominação, seu comportamento corpuscular só foi comprovado em 1929, por Walter Bothe e Werner Kolhörster, na Alemanha [13].

Esta polêmica, porém, pode incluir também o nome de Pacini, uma vez que os experimentos deste foram importantes para definir o caráter não terrestre desta radiação. Registros mais recentes [11] mostram a troca de cartas entre Hess e Pacini, durante o ano de 1920, onde é debatida a contribuição e prioridade de cada um. Alguns autores [8,9 não consideram isto como uma polêmica, tendo em vista que a comunicação entre os dois, apesar de ser cordial, era dificultada pela barreira linguística em um mundo que saía de uma $1^{\mathrm{a}}$ Guerra Mundial, e os sentimentos nacionalistas muitas vezes chegavam à Ciência. Notamos que a questão da prioridade e do pioneirismo na descoberta dos raios cósmicos está mais associada as publicações apenas em língua materna (italiano para Pacini e alemão para Hess) e justifica uma posterior adoção de uma língua franca para a Ciência o inglês.

Pela descoberta da radiação cósmica, Viktor Hess foi agraciado com o Prêmio Nobel de Física em 1936. Domenico Pacini faleceu antes disso, em 1934, devido a uma pneumonia, não tendo assim a honra de ter seus esforços para a descoberta dos raios cósmicos reconhecidos pela Academia Real das Ciências da Suécia.

\subsection{O nascimento de uma nova área da Física}

A descoberta de Hess fez inúmeros cientistas voltarem seus esforços para a recente área dos raios cósmicos, gerando uma grande variedade de resultados: a produção de um efeito cascata ao atingir a atmosfera, estudada por Pierre Auger em 1938, por isto as pesquisas na física dos raios cósmicos se tornou a base para o desenvolvimento da eletrodinâmica quântica e da teoria da cascata eletromagnética 14 15]; a descoberta do méson-pi (píons - nome genérico para $\pi^{-}, \pi^{+}$e $\pi^{0}$ ) por Cesar Lattes, pesquisador brasileiro, em parceria com Giuseppe Occhialini e Cecil Powel, em 1947 [12], confirmando uma previsão teórica feita por Hideki Yukawa em 1935; a proposição de um modelo teórico para os mecanismos de aceleração astrofísicos para explicar como tais partículas chegam a tais energias, feita por Enrico Fermi, em 1949 [12]; bem como a primeira observação de raios cósmicos com energias muito altas, feita por John Linsley, em 1962 [12], entre outros resultados obtidos neste século de observações e medições.

Entre a descoberta dos raios cósmicos e os dias atuais temos uma diferença temporal de mais de 100 anos de grandes avanço neste ramo de pesquisa, devido ao aprimoramento dos aparatos experimentais para detectar os raios cósmicos, seus produtos e subprodutos, assim como modelo teóricos e numéricos para descrever seus processos. Mas ainda há grandes questões a ser resolvidas, como, por exemplo, a origem astrofísica dos raios cósmicos de mais alta energia. Apesar da construção de aceleradores de partículas que acessam uma faixa de energia cada vez mais alta, o estudo dos raios cósmicos ainda é de grande importância na física nuclear e na de física de partículas elementares e campos, por acessar informações sobre partículas e suas interações com energia acima de $10^{17} \mathrm{eV}$ [1], que representa uma energia 5 ordem de grandeza maior do que a alcançada em uma colisão entre prótons no Large Hadron Collider (LHC).

Para esta classe de eventos, denominamos raios cósmicos ultra energéticos (UHECR, acrônimo do inglês Ultra High Energy Cosmic Rays). Eles configuram a grande incógnita a ser resolvida dentro da física dos raios cósmicos, sendo portanto uma área em aberto. Uma de suas questões centrais é a dificuldade de analisar partículas acima de um limiar de energia, igual a $10^{21} \mathrm{eV}$, que representa 16 ordens de grandeza maior que a massa de repouso do eletrón, se seguirmos analisando, um elétron com tal energia, o mesmo deve ter 99,9999999999999999\% da velocidade da luz.

Outra questão que tais raios cósmicos trazem consigo é a sua origem, determinar onde tais raios cósmicos adquirem energia tão elevada. Para compreendermos qual é a provável fonte de tais partículas é necessário ter conhecimento da natureza dos raios cósmicos: sua composição química, seu espectro de energia observado na superfície terrestre e sua distribuição de energia. Outro fator que é necessário na busca por fontes, é o conhecimento sobre como eles se propagam no espaço interestelar e/ou intergaláctico até a Terra, pois essa propagação é responsável pelas mudanças na energia final do mesmo. E por fim o conhecimento da isotropia dos raios cósmicos, pode direcionar a possíveis fontes, sejam elas dentro de nossa galáxia ou fora dela [16].

Os dados obtidos a partir dos raios cósmicos abrangem um grande amontoados de áreas do conhecimento, podendo ser aplicado na Astrofísica, para o estudo do meio interplanetário, ou na Cosmologia, devido aos isótopos cosmogênicos produzidos pelos mesmos.

\section{Fontes de raios cósmicos}

A partir da comprovação de que haveria um emissor de radiação fora do nosso planeta, os olhares se voltaram ao espaço em busca de possíveis fontes. O primeiro candidato foi o Sol, porém a correta determinação da origem dos raios cósmicos por métodos diretos é impossível devido a sua trajetória aleatória causada por suas interações com campos magnéticos turbulentos presentes no espaço.

A partir de métodos indiretos, determinou-se o Sol verdadeiramente como uma fonte de raios cósmicos, os quais geram um fluxo de entrada na atmosfera terrestre relativamente constante. De acordo com sua faixa de energia (até $10^{9} \mathrm{eV}$ ), isso representa menos da metade do espectro da energia medida nos raios cósmicos, denominados por isso de raios cósmicos solares (SCR).

Aproximadamente nesta mesma faixa de energia dos SCR, a partir do estudo da composição química de um 
conjunto de partículas, notou-se que estas não possuem o mesmo mecanismo de aceleração que os raios cósmicos solares, ou dos raios cósmicos galácticos, e por isto este conjunto é denominado de raios cósmicos anômalos (ACR), e o modelo teórico mais aceito sobre seu processo de aceleração trata as mesmas como partículas neutras de gás interestelar que penetram a helioesfera. Por serem neutras, as mesmas não estão sujeitas às forças de Lorentz, restando serem aceleradas por fotoionização ou por trocas com o material do vento solar 17 .

Acima da faixa de energia de $1 \mathrm{GeV}\left(10^{9} \mathrm{eV}\right)$, o Sol não é mais a fonte destes raios cósmicos, portanto devemos expandir a procura de possíveis fontes, e dentro de nossa galáxia encontramos tais fontes. Acredita-se que os raios cósmicos galácticos possam ser acelerados por remanescentes de supernovas, que foi justificado por Ginzburg e Syrovtskii, em 1964 [14], através de argumentos fortes e simples, baseado nos níveis energéticos destes remanescentes. Como estes níveis são altos, se entre 5\% e $10 \%$ da energia cinética dos mesmos for convertida para acelerar raios cósmicos, isso proveria um mecanismo com energia suficiente para todo o espectro de raios cósmicos galácticos [14]. O que torna mais fácil considerar os remanescentes de supernova como fontes é que eles geram um campo magnético com uma intensidade maior do que o meio interestelar. O mecanismo de aceleração destes raios cósmicos é a aceleração estatística, normalmente chamados de mecanismos de Fermi. É bem aceito que partículas com espectro de energia entre $10^{9} \mathrm{eV}$ e $10^{16} \mathrm{eV}$ são aceleradas pelos remanescentes ordinários de supernovas [17.

Se assumirmos uma fonte astrofísica de raio $R$ permeada por um campo magnético de intensidade $B$, conforme uma partícula de carga $q$ se move pelo campo a uma distância $\rho$, descreve uma trajetória circular, através da força centrípeta

$$
\vec{F}_{c p}=\frac{\gamma m_{0} \vec{v}^{2}}{\rho}
$$

mas a força atuante sobre essa partícula é a força de Lorentz

$$
\begin{aligned}
\vec{F}_{c p} & =q \vec{v} \times \vec{B} \\
\frac{\gamma m_{0} v^{2}}{\rho} & =q v B \sin \theta
\end{aligned}
$$

Se a distância $\rho$ for maior que o raio da fonte $R$, a partícula irá sair da região de aceleração, portanto a condição que deve ser satisfeita é a de que $\rho \leq R$. Ao aplicarmos esta condição para o lado esquerdo da (2), temos que

$$
\begin{aligned}
\gamma m_{0} v & \leq q B R \sin \theta \\
p & \leq q B R \sin \theta .
\end{aligned}
$$

Na equação (3) é perceptível que o limite máximo do momentum é determinado quando $\sin \theta=1$, portanto

$$
\begin{aligned}
p_{\max } & \leq q B R \\
E^{\max } & \lesssim c q B R
\end{aligned}
$$

onde utilizamos a aproximação relativística $E \approx p c . \mathrm{A}$ expressão (5) é conhecida como critério de Hillas, sendo uma ferramenta de determinação de possíveis fontes.

Para o caso da Via Láctea, a qual tem um campo magnético de $B=4 \times 10^{6} \mathrm{G}$ e raio de $15 \mathrm{kpc}$. O máximo de energia que seria possivelmente transferida para o mesmo é na ordem $E^{\text {max }} \lesssim 10^{19} \mathrm{eV}$.

Isso implica que raios cósmicos com energias superiores a $10^{19} \mathrm{eV}$ não podem ser acelerados pela Via Láctea. Portanto temos uma expressão para caracterizar possíveis fontes de raios cósmicos, a partir da máxima energia de aceleração possível.

A equação 5 pode ser rearranjada para obter

$$
\begin{aligned}
\frac{E^{\max }}{c q} & \lesssim B R \\
\log \left(\frac{E^{\max }}{c q}\right) & \lesssim \log B+\log R \\
\log B & \lesssim \log R-\log \left(\frac{E^{\max }}{c q}\right),
\end{aligned}
$$

obtendo uma função linear cujo gráfico gerado é denominado Plot de Hillas (Fig,1), que serve como um catálogo para fontes de raios cósmicos, as linhas diagonais representam a energia do raio cósmico acelerado.

Alguns objetos astrofísicos que são possíveis fontes de raios cósmicos são:

- Galáxias com Núcleos Ativos: Galáxias que possuem uma emissão incomum de energia em seu núcleo são denominadas de Galáxias com Núcleos Ativos (AGN - Active Galactic Nuclei), e sua principal característica é que um grande fração de sua luminosidade total não é térmica e predominantemente é emitida pelo núcleo da mesma, a existência de um buraco negro no centro destas possa ser o responsável por tal emissão.

Podem haver AGNs de várias classes, pois há vários fatores distintos sobre as mesmas, como a emissão de espectros em diferentes comprimentos de onda e a presença ou não de jatos. Alguns casos são: Quasares e Rádio-galáxias.

- Estrelas de Nêutrons: Objetos de interesse como um mecanismo de aceleração de UHECR como pode ser visto no diagrama de Hillas (Fig,1). Apesar de seu tamanho pequeno, a presença de um forte campo magnético lhe dá a possibilidade de acelerar prótons a energia de $10^{20} \mathrm{eV}$. Um classe destas estrelas são os pulsares, que são fortemente magnetizados e em rotação.

- Explosões de Raios- $\gamma$ : As explosões de raios- $\gamma$ (GRB - Gamma-Ray Burts) podem emitir partículas carregadas e até mesmo neutrinos com energias muito 


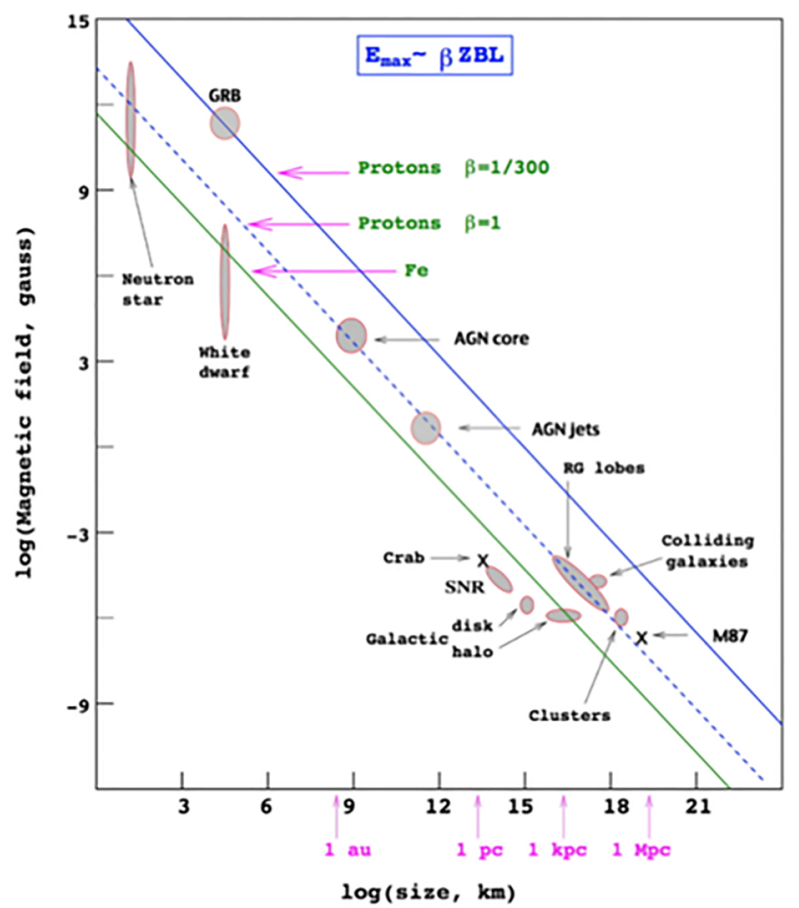

Figura 1: Diagrama de Hillas, diagrama que conecta o campo magnético e o tamanho da região de aceleração, os objetos que estão abaixo da linha verde não são capazes de acelerar prótons ou núcleons de ferro a energias elevadas, portanto, os tornando não fontes de UHECR.

elevadas, tornando tais objetos astrofísicos como possíveis fontes de raios cósmicos.

Para determinar-se se o candidato a fonte de raios cósmicos é adequado, é necessário determinar a direção de chegada dos raios cósmicos da faixa de energia de interesse apontando ao respectivo candidato. Por exemplo, no caso da supernova, onde o modelo de aceleração é uma onda de choque, as partículas carregadas terão alteração no seu percurso, tanto no momento da aceleração quanto em sua propagação, por este motivo que há uma importância maior na análise de componentes neutros, como os raios- $\gamma$ ou os neutrinos. Os modelos que melhor descrevem raios- $\gamma$ não são hadrônico $\aleph^{1}$ e sim eletromagnéticos, e baseiam-se na aceleração de elétrons e no processo Compton inverso.

\section{Propagação}

Após a partícula sair da sua fonte, fica a questão: como ela chega até nós? Se a partícula é produzida na nossa galáxia, ela deve atravessar o meio interestelar para chegar à Terra. Se a origem da mesma for extragaláctico, ela deve atravessar o meio interestelar da sua galáxia de origem, cruzar o meio intergaláctico até o meio interestelar da nossa galáxia, atravessando o mesmo até atingir a Terra.

\footnotetext{
${ }^{1}$ Apesar de não termos falado sobre os hádrons ainda, basicamente são as partículas que são afetadas pela interação nuclear forte, e são compostas por quarks, ligados por glúons.
}

Primeiramente consideramos que o meio interestelar é composto por nuvens de gases tanto neutras como ionizados, predominantemente hidrogênio, ionizado pela luz das estrelas. Outras componentes que influenciam na propagação do raio cósmico incluem o campo magnético galáctico caótico e o regular, e a radiação cósmica de fundo (CBM - Cosmic Microwave Background) a 2, $7 \mathrm{~K}$ provenientes do Big Bang [16]. O campo galáctico regular tem uma força de $(1 \sim 3) \times 10^{-6} \mathrm{~T}$, o mesmo está inserido no plano galáctico, com latitude galáctica de $90^{\circ}$.

Os campos caóticos são produzidos em nuvens magnéticas geradas devido ao movimento de transmissão dos gases ionizados. As magnitudes de ambos os campos são de mesma ordem [16]. A trajetória do raio cósmico é modificada e espalhada pelos campos regulares e caóticos, através de interações eletromagnéticas, isto produz um movimento difusivo, espalhado, de raios cósmicos na galáxia.

Em movimento difuso, prótons e elétrons se propagam através da matéria galáctica e vão sofrendo interações com o meio, e a partir disto produzir raios- $\gamma$,e outros subprodutos, através de interações eletromagnéticas. O comprimento característico para a deflexão magnética é dada pelo raio de Larmor.

$$
R_{L}=\frac{E}{300(H Z)}
$$

onde $Z$ é o número atômico e $R_{L}$ é dado em centímetros, $E$, em eV, e $H$ em Gauss. Raios cósmicos com energias até a ordem de $10^{15} \mathrm{eV}$ podem ficar enrolados nas nuvens magnéticas e sofrer uma grande deflexão. Esta deflexão depende do comprimento da nuvem magnética $\ell$,dado em parsecs, sendo que o ângulo de deflexão é dado por $\frac{\ell}{R_{L}}$.

Estas partículas serão difundidas através da galáxia com o livre caminho médio igual à distância média entre nuvens magnéticas. Para maiores energias o ângulo de deflexão é menor, pois $R_{L}$ cresce com a energia e o ângulo é proporcional a $\frac{1}{R_{L}}$. Ainda assim, um número alto de tais dispersões podem produzir um grande deflexão. Para $N$ colisões independentes, a deflexão angular total irá ser grande quando:

$$
N \approx\left(\frac{E}{300(H Z) l}\right)^{2}
$$

Como $R_{L} \propto E$, o número de colisões necessárias para aumentar a deflexão angular cresce com o quadrado da energia $E^{2}$. Isto implica que o livre caminho médio é também proporcional a $E^{2}$ e o movimento é não difusivo. Como regra geral, o efeito de campos magnéticos caóticos é pequeno para partículas com energia a partir de $10^{18}$ $\mathrm{eV}$.

$\mathrm{Na}$ faixa de energia de $10^{15} \mathrm{eV}$ a difusão é importante. Isto significa que o tempo que cada raio cósmico permanece na galáxia (sua idade), $t_{D}$, é maior do que para 
propagações sem difusão, $t$. A teoria da difusão nos dá um acréscimo relativo de

$$
\frac{t_{D}}{t} \approx \frac{1}{2\left(\frac{r}{\lambda}\right)}
$$

onde $r$ é a distância da fonte até o limite da região de difusão e $\lambda$ é livre caminho médio. A quantidade de gás cósmico que o raio cósmico atravessa é proporcional a $\frac{r}{\lambda}$. Se uma partícula é originária do centro da galáxia e é difundida até a terra, ela atravessa $\leq 10 \mathrm{~g} / \mathrm{cm}^{2}$ de matéria. Desde de que o comprimento de interação de prótons a energia $10^{15} \mathrm{eV}$ é $70 \mathrm{~g} / \mathrm{cm}^{2}$, o espectro do próton não será afetado pela difusão. O livre caminho médio para a espalaçã $\coprod^{2}$ de núcleos pesados é muito menor, em ordem de $10 \mathrm{~g} / \mathrm{cm}^{2}$. Isto nos leva a esperar que a composição química do fluxo de raios cósmicos será alterada pelo seu movimento de difusão.

Isso é reafirmado quando se analisa a composição química dos raios cósmicos observados, conforme observamos na Fig (2), onde conclui-se que há uma abundância de elementos químicos que não se tem em tanta abundância na Terra e nem em nosso sistema solar.

Enquanto a maioria dos grupos tem a mesma abundância relativa, há certo grupos de elementos que estão em maior abundância no raios cósmicos por várias ordens de grandeza, o primeiro grupo formado pelos elementos Ti, Sc, B, Be, Li. A razão para abundância de tais elementos se da a partir da interação do raios cósmico com a matéria galáctica e a partir da espalação, resultado da quebra de um núcleo pesado primário, portanto esses elementos são conhecidos como elementos secundários na composição dos raios cósmicos.

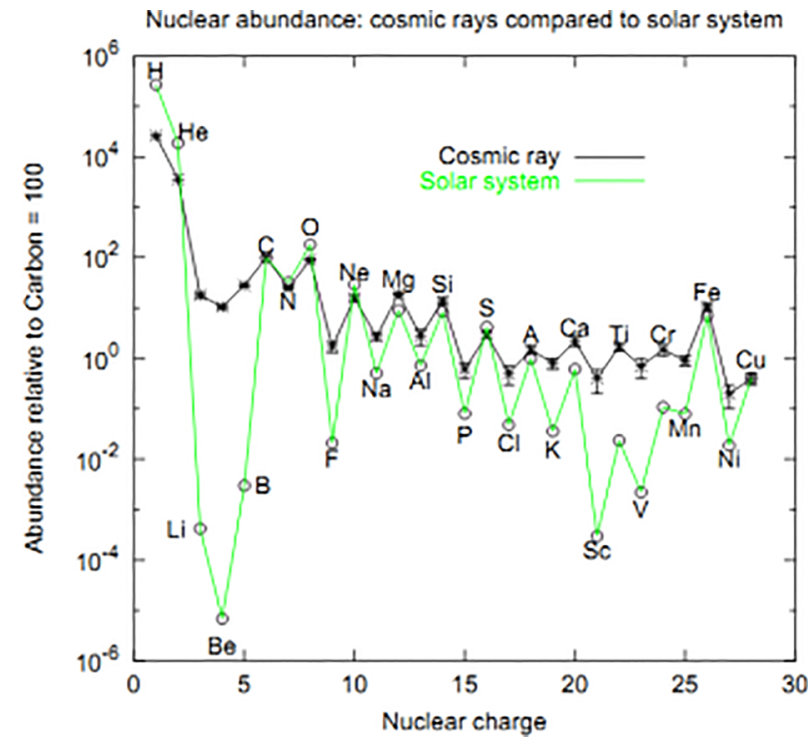

Figura 2: Comparação entre a abundância de elementos entre o sistema solar e os elementos observados no raios cósmicos.

${ }^{2}$ Reação nuclear natural que ocorre causando a nucleossíntese, que é o processo de criação de novos núcleos atômicos a partir dos núcleos pré-existentes (prótons e nêutrons)
Na composição química dos raios cósmicos de baixa energia $\left(10^{12} \sim 10^{15} \mathrm{eV}\right)$ predominam prótons que representam aproximadamente $60 \%$, e partículas $\alpha$ constituem $25 \%$, núcleos de N, C, O e Fe que representam $15 \%$ e restante é composto por elétrons, $\leq 10^{-2} \%$, e raios- $\gamma$, na ordem $10^{-3} \%$ do fluxo de raios cósmicos. Ao analisarmos em altas energias, como visto o livre caminho médio se torna proporcional a $\mathrm{E}^{2}$ o que implica que $\frac{r}{\lambda}$ decresce e o tempo de difusão se aproxima do tempo sem difusão.

O aparecimento de elétrons no espectro de raios cósmicos é pouco porque eles são efetivamente absorvidos pelo espalhamento Compton com os fótons e pelo processo bremsstrahlung (freamento de elétrons), com a taxa de perda de energia, $\frac{d E}{d x} \propto E^{2}$. Assim, haverá muito poucos elétrons altamente energéticos, e o processo de perda de energia se torna um gerador continuo de fótons de baixa energia, os quais podem ser observados, indicando o espectro dos elétrons. Outro fator que contribui para o não aparecimento de elétrons em abundância na composição dos raios cósmicos, é o fato de haver perda de energia pela radiação síncrotron ${ }^{3}$ O elétron interage com fortes campos magnéticos ao mesmo tempo que é acelerado, assim o mesmo perde energia.

O meio intergaláctico é modelado como apresentando um campo magnético na ordem de $10^{-2}$ do campo galáctico. Partículas do centro do superaglomerado local não serão significativamente desviados para energias acima de $3 \times 10^{19} \mathrm{eV}$. Podemos portanto esperar uma anisotropia para fontes extragalácticas relativamente próximas para energias altas. Para grandes distâncias essa informação direcional infelizmente acaba se perdendo. O tempo de vida estimado para raios cósmicos em super agrupamentos locais é da ordem $10^{10}$ anos.

\subsection{Interações Fóton-Núcleo (raio cósmico)}

Em meio a todo esse trajeto intergaláctico e estelar, o raio cósmico, seja ele, um núcleo, ou até mesmo um fóton altamente energético, raios- $\gamma$, interage com os componentes deste semi-vácuo que é o meio cósmico. Um dos tipos de interações possíveis, são as interações Fóton-Núcleo.

Para calcularmos tais interações temos primeiro que calcular o limiar de energia 4 Para isto consideremos a interação de duas partículas $a$ e $b$, criando duas novas partículas $c$ e $d$ no referencial de Laboratórid 5

$$
a+b \rightarrow c+d
$$

\footnotetext{
${ }^{3}$ Radiação eletromagnética gerada por partículas portadoras de carga, elétrons, $e^{-}$, se movendo com velocidade relativística ao longo de trajetórias curvas.

${ }^{4} \mathrm{Na}$ física de partículas, o limiar de energia para a produção de uma partícula é a energia cinética mínima que um par de partículas em movimento deve ter quando colidir.

${ }^{5}$ No referencial de laboratório temos a partícula denominada de projétil que pode ser descria por $a$, e a partícula denominada de alvo que pode ser descrita por $b$. Portanto temos que para o referencial de laboratório $\vec{p}_{b}=0$.
} 
onde as partículas tem massa $m_{a}$ e $m_{b}$, e quadrimoment ${ }^{6}$ $P_{a}^{\mu}=\left(E_{a}, \vec{p}_{a}\right)$ e $P_{b}^{\mu}=\left(E_{b}, \vec{p}_{b}\right)$. Realiza-se a contração entre os quadrimomentos em cada um dos referenciais, centro de massa e de laboratório,$P^{\mu} P_{\mu}=P^{\prime \mu} P_{\prime_{\mu}}$, onde o quadrimomento com o índice linha representa o referencial do centro de massa 7 já o sem linha representa o referencial do laboratório. Como $P^{\mu}=P_{a}^{\mu}+P_{b}^{\mu}$, a contração é

$$
\begin{aligned}
P^{\mu} P_{\mu} & =P^{\prime \mu} P_{\mu}^{\prime} \\
\left(E_{a}+E_{b}\right)^{2}-\left(\vec{p}_{a}+\vec{p}_{b}\right)^{2} & =\left(E_{a}^{\prime}+E_{b}^{\prime}\right)^{2}-\left(\vec{p}_{a}^{\prime}+\vec{p}_{b}^{\prime}\right)^{2}
\end{aligned}
$$

No referencial do centro de massa, temos que $\vec{p}_{a}^{\prime}=-\vec{p}_{b}$, portanto 11 fica

$$
\begin{aligned}
\left(P_{a}^{\mu}+P_{b}^{\mu}\right)^{2} & =E^{\prime 2} \\
E^{\prime 2} & =P_{a}^{\mu} P_{\mu_{a}}+P_{b}^{\mu} P_{\mu_{b}}+2 E_{a} E_{b}-2 \vec{p}_{a} \cdot \vec{p}_{b}
\end{aligned}
$$

Como $P_{a}^{\mu} P_{\mu_{a}}=m_{a}^{2}$ e $P_{b}^{\mu} P_{\mu_{b}}=m_{b}^{2}$, temos que

$$
E^{\prime 2}=m_{a}^{2}+m_{b}^{2}+2 E_{a} E_{b}-2 \vec{p}_{a} \cdot \vec{p}_{b}
$$

Seja a energia necessária para a criação das partículas descritas no processo (9),

$$
E_{\text {min }}^{\prime}=m_{a}+m_{b}+\Delta m=m_{c}+m_{d}
$$

onde $\Delta m$ é a diferença de massa que foi utilizada ou gasta ao longo do processo inelástico. Relacionando as equações 114 e 15

$$
\begin{aligned}
E^{\prime 2} & =E_{\text {min }}^{\prime 2} \\
m_{a}^{2}+m_{b}^{2}+2 E_{a} E_{b}-2 \vec{p}_{a} \cdot \vec{p}_{b} & =E_{\text {min }}^{2} \\
E_{a} E_{b}-\vec{p}_{a} \cdot \vec{p}_{b} & =\frac{1}{2}\left[E_{\text {min }}^{\prime 2}-m_{a}^{2}-m_{b}^{2}\right]
\end{aligned}
$$

Substituindo 15 ,

$$
\begin{aligned}
& E_{a} E_{b}-\vec{p}_{a} \cdot \vec{p}_{b}=\frac{1}{2}\left[\left(m_{a}+m_{b}+\Delta m\right)^{2}-m_{a}^{2}-m_{b}^{2}\right] \\
& E_{a} E_{b}-\vec{p}_{a} \cdot \vec{p}_{b}=\frac{1}{2}\left[2 m_{a} m_{b}+\Delta m^{2}+2 \Delta m\left(m_{a}+m_{b}\right)\right] \\
& E_{a} E_{b}-\vec{p}_{a} \cdot \vec{p}_{b}=m_{a} m_{b}+\Delta m\left(m_{a}+m_{b}+\frac{\Delta m}{2}\right)
\end{aligned}
$$

$\overline{{ }^{6} \text { Estamos usando unidades naturais, ou seja, } \hbar=c=1}$

${ }^{7}$ No referencial de centro de massa, ambas as partículas estão convergindo uma para a outra cominando na colisão, portanto temos que

$$
\vec{p}_{a}+\vec{p}_{b}=0
$$

Para o referencial de laboratório temos que $\vec{p}_{b}=0$, a eq. 21 fica

$$
E_{a} E_{b}=m_{a} m_{b}+\Delta m\left(m_{a}+m_{b}+\frac{\Delta m}{2}\right)
$$

Fotoprodução de pares: Para o caso onde a interação ser entre um núcleo e um fóton da radiação cósmica de fundo, CMB, gerando como produto um par elétron-pósitron, $e^{-} e^{+}$, esse processo que é denominado como fotoprodução de pares. Podemos representar a interação como

$$
A+\gamma_{C M B} \rightarrow A+e^{+}+e^{-}
$$

Como a interação é no referencial de laboratório, e a massa do fóton $m_{\gamma}=0$, a expressão para esse processo se torna, no ref. de laboratório,

$$
E_{A} E_{\gamma_{C M B}}=\Delta m\left(m_{A}+\frac{\Delta m}{2}\right)
$$

A diferença $\Delta m$ para este processo é dada por

$$
\Delta m=m_{e}+m_{e}+m_{A}-m_{A}-m_{\gamma_{C M B}}=2 m_{e}
$$

onde a massa do núcleo $m_{A}$ dada por $m_{A} \approx A m_{p}$. Como a energia do fóton da CMB é de $\approx 2,34 \times 10^{-4} \mathrm{eV}$, usando

$$
E_{A}=\frac{m_{e}}{E_{\gamma_{C M B}}}\left(A m_{p}+m_{e}\right)
$$

e $m_{p}=1836 m_{e}$, temos

$$
E_{A}=2,2 \mathrm{GeV}(A \cdot 1836+1)
$$

Para o caso de um próton, ou seja, $A=1$, temos que a miníma energia necessária é

$$
E_{p} \approx 10^{12} \mathrm{eV}
$$

Para o caso de A ser um núcleo pesado, assim como o Ferro $F e, A=26$ e miníma energia é

$$
E_{F e} \approx 10^{14} \mathrm{eV}
$$

Fotoprodução de píons Para o caso onde a interação entre um Núcleo e um fóton gere como produto l's Píons neutros 8

$$
A+\gamma_{C M B} \rightarrow A+l \pi^{0}
$$

No referencial de laboratório, a expressão para esse processo se torna

${ }^{8} \mathrm{O}$ méson $\pi^{0}$ é constituído pelo par quark anti-quark, e é instável,
com tempo de vida média da ordem de $10^{-16}$ segundos. Na descri-
ção d eYukawa, este méson é um dos mediadores da força nuclear forte. 


$$
E_{A} E_{\gamma_{C M B}}=\Delta m\left(m_{A}+\frac{\Delta m}{2}\right)
$$

Sejam

$$
\left\{\begin{aligned}
m_{A} & =A m_{p} \\
\Delta m & =l m_{\pi^{0}} \\
m_{\pi^{0}} & \approx 135 \mathrm{MeV} \\
m_{p} & \approx 6,95 m_{\pi^{0}}
\end{aligned}\right.
$$

Temos que a eq.30, ficará

$$
E_{A} \approx 5 \times 10^{20} \mathrm{eV} l\left(A+\frac{l}{2}\right)
$$

Para o caso do próton interagindo com um fóton da radiação cósmica de fundo, $\mathrm{CBM}$, produzindo um píon, temos que $A=1$ e $l=1$, cai-se no corte GZK, que irá ser abordado em mais detalhes. Para o caso de termos um núcleo de Ferro $F e, A=26$ e a miníma energia que o Fóton precisa para produzir a reação 29 com $l=1$, é de $E_{\gamma} \approx 135 \mathrm{MeV}$.

\subsection{Corte GZK}

Na década de 60, em Volcano ranch, fazenda perto de Albuquerque, no Novo México-EUA. Foi detectado o primeiro raio cósmico ultra-energético, com energia na ordem de Zeta eletron-voltz, $10^{21} \mathrm{eV}$, sendo que há detecções de partículas com energia acima desta ordem até o tempo presente, mais de 50 anos após. Uma explicação para não haver detecção de raios cósmicos de ordem mais alta está fortemente conectado com o efeito da fotoprodução de píons que ocorre ao londo da propagação do raio cósmico. A explicação, corte, foi proposto também na década de 60, denominado de Corte $G Z K$, devido ao nome dos pesquisadores que o propuseram, Greisen-ZatsepinKuzmin. Prótons com energia na ordem de $5 \times 10^{19} \mathrm{eV}$ irão colidir inelasticamente com os fóton $\$^{9}$ da radiação cósmica de fundo (CMB) que tem uma densidade de 500 fótons $/ \mathrm{cm}^{3}$ e produto destas colisões serão uma ressonância que irá decair em um próton de mais baixa energia e em um píon neutro

$$
\gamma^{C M B}+p \rightarrow \Delta^{+} \rightarrow p+\pi^{0}
$$

com energia na ordem de $300 \mathrm{MeV}$, o que corresponde ao limite para fotoprodução de píons $\approx 7 \times 10^{19} \mathrm{eV}$. Por causa do inicio dessas interações inelásticas, na propagação a energias na ordem de $5 \times 10^{19} \mathrm{eV}$, se espera um declive ou corte no fluxo de raios cósmicos a partir dessa energia, o que é denominado de corte GZK (GreisenZatsepin-Kuzmin) 2, 3, proposto nos anos 1960.

Para construirmos o calculo do corte, desprezamos a ressonância ${ }^{10} \Delta^{+}$, devido a seu tempo de vida curto,

${ }^{9}$ Fótons de energia de $2,34 \times 10^{-4} \mathrm{eV}$, a uma temperatura de 2,7 K.

${ }^{10}$ Bárion Delta ou Ressonância Delta, é um hádron instável, constituído por 3 quarks, up e downs. O mesmo possui carga elétrica, $+1 e$, com tempo de vida médio na ordem de $10^{-24}$ segundos
$10^{-24}$, e portanto analisamos o produto do seu decaimento,

$$
\gamma^{C M B}+p \rightarrow p+\pi^{0}
$$

Em unidades naturais ${ }^{11}$ temos o quadrimomento $P^{\mu}=$ $(E, \vec{p})$ e a energia relativística quadrática $E^{2}=m^{2}+p^{2}$, e da conservação de energia obtemos

$$
P_{i}^{\mu}=P_{f}^{\mu}
$$

No referencial do centro de massa, se tem que o momento linear e a energia da colisão apresentada em (34) será tal qual

$$
\left\{\begin{array}{l}
\vec{p}_{p_{i}}+\vec{p}_{\gamma}^{\prime}=0=\vec{p}_{p_{f}}+\vec{p}_{\pi} \\
E_{p_{i}}^{\prime}+E_{\gamma}^{\prime}=E_{p_{f}}^{\prime}+E_{\pi}^{\prime}
\end{array}\right.
$$

A energia mínima para que ocorra a produção do píon é que sua energia de repouso esteja disponível no sistema, portanto $\vec{p}_{\pi}=0$ e a energia total deste píon é igual a sua massa

$$
E_{\pi_{\text {min }}}^{\prime}=m_{\pi}
$$

e o mesmo é válido para o próton após a colisão, portanto podemos afirmar que $\vec{p}_{p_{f}}=0 \mathrm{e}$

$$
E_{p_{f}}^{\prime}=m_{p}
$$

Por isso, podemos reescrever 35 como

$$
\left\{\begin{array}{l}
\vec{p}_{p_{i}}^{\prime}+\vec{p}_{\gamma}^{\prime}=0=\vec{p}_{p_{f}}+\vec{p}_{\pi} \\
E_{p_{i}}^{\prime}+E_{\gamma}^{\prime}=m_{p}+m_{\pi}
\end{array}\right.
$$

Lembrando que a contração $P^{\mu} P_{\mu}$ é um invariante de Lorentz, podemos calcular esta quantidade para cada referencial

- Para o centro de massa

$$
\begin{aligned}
& P^{\prime \mu} P_{\mu}^{\prime}=\left(P_{p_{i}}^{\prime \mu}+P_{\gamma}^{\prime \mu}\right)^{2} \\
& P^{\prime \mu} P_{\mu}^{\prime}=\left(E_{p_{i}}^{\prime}+E_{\gamma}^{\prime}\right)^{2}-\left(\vec{p}_{p_{i}}^{\prime}+\vec{p}_{\gamma}^{\prime}\right)^{2}
\end{aligned}
$$

Daí, usando 38 , temos

$$
P^{\prime} \mu P_{\mu}^{\prime}=\left(m_{p_{i}}+m_{\gamma}\right)^{2}
$$

- Para o referencial do laboratório

$$
\begin{aligned}
P^{\mu} P_{\mu} & =\left(P_{p_{i}}^{\mu}+P_{\gamma}^{\mu}\right)^{2} \\
P^{\mu} P_{\mu} & =\left(E_{p_{i}}+E_{\gamma}\right)^{2}-\left(\vec{p}_{p_{i}}+\vec{p}_{\gamma}\right)^{2} \\
P^{\mu} P_{\mu} & =E_{p_{i}}^{2}+E_{\gamma}^{2}+2 E_{p_{i}} E_{\gamma}-\vec{p}_{p_{i}}^{2} \\
& -\vec{p}_{\gamma}^{2}-2 \vec{p}_{p_{i}} \cdot \vec{p}_{\gamma}
\end{aligned}
$$


As duas expressões devem trazer a mesma informação física, pois são invariantes $P^{\mu} P_{\mu}=P^{\prime} \mu P_{\mu}^{\prime}$. Então

$$
\begin{aligned}
& \left(E_{p_{i}}^{2}+E_{\gamma}^{2}+2 E_{p_{i}} E_{\gamma}-\vec{p}_{p_{i}}^{2}-\vec{p}_{\gamma}^{2}-2 \vec{p}_{p_{i}} \cdot \vec{p}_{\gamma}\right)_{L a b} \\
& =\left(m_{p}+m_{\pi}\right)_{C M}^{2},
\end{aligned}
$$

mas, como $m_{\gamma}=0$, temos

$$
\begin{cases}E_{p_{i}}^{2} & =m_{p}^{2}+p_{p_{i}}^{2} \\ E_{\gamma}^{2} & =p_{\gamma}^{2}\end{cases}
$$

Substituindo este resultado em (43)

$$
\begin{aligned}
& m_{p}^{2}+p_{p_{i}}^{2}+p_{\gamma}^{2}+2 E_{p_{i}} E_{\gamma}-\vec{p}_{p_{i}}^{2}-\vec{p}_{\gamma}^{2}-2 \vec{p}_{p_{i}} \cdot \vec{p}_{\gamma} \\
& =m_{p}^{2}+m_{\pi}^{2}+2 m_{p} m_{\pi} \\
& 2 E_{p_{i}} E_{\gamma}-2 \vec{p}_{p_{i}} \cdot \vec{p}_{\gamma}=m_{\pi}^{2}+2 m_{p} m_{\pi}
\end{aligned}
$$

O produto escalar entre os momento lineares do próton incidente e do fóton CMB é dada em termos do ângulo $\theta$ entre os momentos

$$
\vec{p}_{p_{i}} \cdot \vec{p}_{\gamma}=\left|\vec{p}_{p_{i}}\right|\left|\vec{p}_{\gamma}\right| \cos \theta
$$

Usando as relações apresentadas em (44), obtemos

$$
\vec{p}_{p_{i}} \cdot \vec{p}_{\gamma}=E_{\gamma} \sqrt{E_{p_{i}}^{2}-m_{p}^{2}} \cos \theta
$$

Considerando que o próton tem uma energia cinética muito maior que sua massa de repouso $\left(E_{p_{i}}^{2} \gg m_{p}^{2}\right)$ devido ao fato de que essa previsão é descrita para partículas ultra relativísticas, logo $\sqrt{E_{p_{i}}^{2}-m_{p}^{2}} \approx E_{p_{i}}$, resultando que Eq. 47 é simplificada como

$$
\vec{p}_{p_{i}} \cdot \vec{p}_{\gamma}=E_{\gamma} E_{p_{i}} \cos \theta
$$

Retornando este resultado em 45 , chegamos à

$$
E_{p_{i}}=\frac{m_{\pi}\left(2 m_{p}+m_{\pi}\right)}{2 E_{\gamma}(1-\cos \theta)}
$$

A energia mínima que o próton incidente deverá ter para que a reação ocorra será dada quando $\cos \theta=-1$, quando a expressão (49) obterá o seu menor valor possível. Neste caso, temos

$$
\left(E_{p_{i}}\right)_{\min }=\frac{m_{\pi}\left(2 m_{p}+m_{\pi}\right)}{4 E_{\gamma}}
$$

Substituindo os valores tabelados, $E_{\gamma}=2,34 \times 10^{-4}$ $\mathrm{eV}, m_{p}=938,26 \mathrm{MeV}$ e $m_{\pi}=134,96 \mathrm{MeV}$, obtemos que este valor mínimo de energia para o próton incidente é

$$
\left(E_{p_{i}}\right)_{\min }=2,925 \times 10^{20} \mathrm{eV}
$$

Portanto, somente prótons com energia acima da ordem $10^{20} \mathrm{eV}$ irá interagir com os fótons da CMB a ponto de produzir píons e como consequência diminuir a sua energia, essa previsão é usada para explicar o motivo de não detectar-se raios cósmicos com ordem de energia maior do que $10^{20} \mathrm{eV}$.

Quaisquer que sejam as fontes dos raios cósmicos extragalácticos, esta interação entre os mesmos e a radiação cósmica de fundo irá produzir um fluxo de neutrinos. Considerando que o ponto desta interação está localizado em escala cosmológica da Terra, estes neutrinos produzidos podem ser rastreados ao ponto com excelente precisão 19]. Estes neutrinos GZK podem ter seu fluxo calculado na sequência do cálculo acima, sendo que sua magnitude é determinada por sua densidade total de energia no Universo.

\section{Raios cósmicos na Terra}

Ao fim de todo seu trajeto no meio celeste, o raio cósmico encontra em seu caminho um pequeno planeta com uma atmosfera que propicia a vida em forma de carbono, a qual consegue detectar estes raios cósmicos e formar um espectro da chegada dos mesmos.

Quando a partícula altamente energética que compõem o raio cósmico adentra a atmosfera é como se a mesma estivesse em uma mesa de bilhar cheia de bolinhas, ela irá colidir com os núcleos presentes em nossa atmosfera e isso gerará certo decaimentos e ionizações e até mesmo criação de certas partículas. A detecção dos raios cósmicos pode ser feito de forma direta, assim como era feito nos primórdios da física dos raios cósmicos com Hess, mas para isso necessita-se que haja um grande fluxo de raios cósmicos com uma determinada energia e temos que desprezar que há interações ao longo do trajeto do topo da atmosfera até o nível do mar.

O estudo de raios cósmicos com energia um pouco a cima de $10^{15} \mathrm{eV}$ deve ser indireto devido ao baixo fluxo envolvido. Os dados experimentais advêm a partir da detecção e da amostragem de chuveiros atmosféricos extensos (EAS - Extensive Air Shower) produzidos pelo raio cósmico conforme o mesmo interage com a atmosfera, os quais abordaremos em mais detalhes ao longo do capítulo.

O chuveiro atmosférico extenso se divide em algumas componentes e modelos, entre suas componentes, temos a parte eletromagnética. A partir da medição dos raios cósmicos, é possível montar um espectro da chega dos mesmos, da pela Fig.3 o espectro obedece uma lei de potência assim como foi visto no capítulo anterior, onde os mecanismos de Fermi geram um espectro que obedece uma lei de potência.

$$
\frac{\mathrm{d} N}{\mathrm{~d} E} \sim E^{-\alpha}
$$

Como pode ser visto na Fig, 3 o fluxo de raios cósmicos tem uma grande variação entre $10^{7} \mathrm{eV}(10 \mathrm{MeV})$ a $10^{21} \mathrm{eV}(10 \mathrm{EeV})$, raios cósmicos com energia da ordem de $10^{17}$ tem um fluxo de $1\left[\frac{\text { próton }}{\mathrm{m}^{2} \mathrm{sMeV}}\right]$ enquanto que os raios 


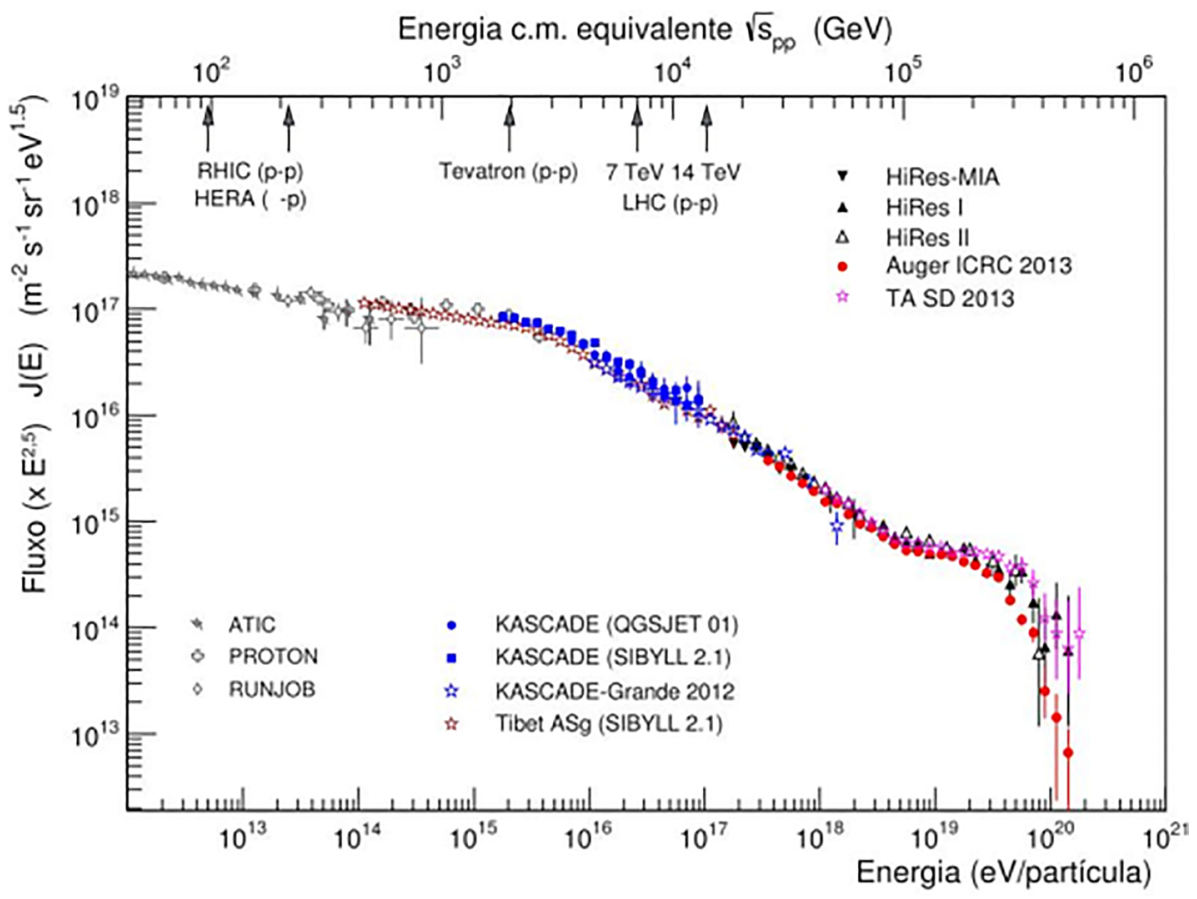

Figura 3: Fluxo de raios cósmicos atingindo a Terra, reescalado pela energia à potência de 2,5. No eixo superior, energia equivalente no centro de massa de uma colisão próton-próton.

cósmicos com energia na faixa de $10^{21} \mathrm{eV}$ tem um fluxo de $1\left[\frac{\text { próton }}{\mathrm{Km}^{2} \mathrm{sMeV}}\right]$

$\mathrm{O}$ valor $\alpha$ presente na expressão 52 indica o índice espectral que é aproximadamente 2,7. Apesar de o espectro caracterizar-se por uma lei de potência praticamente fixa, é perceptível a partir da Fig.3 3 observar que há uma certa mudança de comportamento no espectro para diferentes faixas de energias. Essas mudanças no índice espectral estão estritamente conectadas com os tópicos abordados anteriormente ao longo do texto, ou seja, onde o raio cósmico é gerado e como ele faz a sua viagem até a nossa percepção são os influenciadores no espectro. E o mesmo serve como um verificador de tais casos podem parecer um tanto quanto fictício.

A primeira mudança da inclinação na Fig 3 está na faixa de energia de $3 \times 10^{15} \mathrm{eV}$ e é denominado de primeiro joelho, a mudança torna o espectro mais inclinado e o índice espectral vai de 2,7 para 3,1 . Na faixa de energia de $10^{17} \mathrm{eV}$ temos uma nova mudança na inclinação do espectro e novamente ele se torna mais inclinado, onde seu índice espectral vai de 3,1 para 3,3 , essa mudança é denominada de segundo joelho. O conjunto destas duas mudanças representa o Joelho (knee). A última mudança no espectro é chamada de tornozelo (ankle), onde a mesma retorna o índice espectral novamente para algo próximo a 2,7, o tornozelo se encontra na faixa de energia de $10^{19} \mathrm{eV}$, e representa a parte final do espectro até então.

Como pode ser visto a ordenada da Fig. 3 representa o fluxo, e para baixas energias, abscissa, o fluxo de raios cósmicos tem um valor elevado. Elevado o suficiente para que seja possível se fazer medidas a partir de balóes, vide o trabalho feito por Hess 7, que nos proporcionou a possibilidade de detectar os primeiros raios cósmicos. Portanto, esse alto fluxo possibilita a detecção direta que possibilita o estudo da composição química dos mesmos, assim como foi discutido no capítulo anterior.

Há fortes evidências de que os raios cósmicos que tem sua origem em nossa galáxia, sejam os que estão na faixa do espectro até a região do joelho, entro primeiro e o segundo, sendo os mesmos acelerados a partir dos mecanismos de Fermi, de primeira ou segunda ordem, e como pode ser calculado os mecanismos de Fermi geram um espectro de Lei de Potência, o que corrobora com o espectro analisado.

O Joelho é relacionado com uma variação na composição dos raios cósmicos, experimento reportam um aumento na proporção Ferro e outros núcleos pesadas nesta faixa do espectro, a melhor interpretação para tal efeito pode ser dado pelo modelo galáctico denominado de Leaky Box, onde o modelo permita uma difusão livre dos raios cósmicos com disco galáctico, em alguns exemplos, até mesmo com o halo galáctic ${ }^{12}$. Assumi-se que as partícula são refletidas conforme se aproximam da borda galáctica, sendo assim, ficam aprisionadas na borda galáctica com uma certa probabilidade de saida. Esse modelo tem uma forte correlação com o Raio de Larmor. E a consequência deste modelo é o confinamento de prótons no halo e tornando a composição dos raios cósmicos nesta faixa de energia mais voltada para o núcleo pesados 16.

\footnotetext{
${ }^{12} \mathrm{O}$ entorno da galáxia
} 
Quando adentramos na faixa de energia de $10^{19} \mathrm{eV}$, estamos tratando do tornozelo do espectro, para energias deste valor e acima o campo magnético galáctico não tem mais como aprisionar quaisquer partícula, nem mesmos núcleos pesados. Por exemplo, para um próton de energia na ordem $10^{19} \mathrm{eV}$ o raio de Larmor será de $10 \mathrm{Kpc}$, que é maior do que as dimensões da galáxia, e em nossa galáxia não se tem nenhum objeto astrofísico capaz de acelerar um raio cósmico a tal energia, eis então que a mudança no espectro que o tornozelo causa é dita como sendo a transição dos raios cósmicos galácticos para os raios cósmicos extra galácticos e isto causa um certo achatamento no espectro. E traz consigo um grande questionamento, há a existência do corte GZK ou não? Bom, tal questionamento nos leva a três possíveis cenários.

1. O espectro continua indefinitivamente com o mesmo comportamento que vinha apresentado abaixo de $10^{19} \mathrm{eV}$, o que não está de acordo com o dados obtidos, este cenário implicaria que as fontes deste raios cósmicos sejam próximas, ou seja, as fontes devem ser galácticas ou extragalácticas a uma distância de poucos mega parsecs de distância. O fluxo deve ser predominantemente de prótons, isso desenvolveria um anisotopria em direção ao plano galáctico ou em direção a sua fonte extragaláctica.

2. O corte GZK é observado na faixa de $\mathrm{EeV}, 10^{18} \mathrm{eV}$, isto implica que a fonte está a mais de $20 \mathrm{Mpc}$, e consequentemente a fonte é extragaláctica, medições de anisotropia daria um indicativo de onde tal raio cósmico provêm.

3. Para energia acima de $10^{19} \mathrm{eV}$ o espectro continua mas com o perfil regido pelo índice espectral do tronozelo. Esse cenário é justificado se correlacionarmos o fluxo de raios cósmicos presentes nessa faixa de energia devidos ao Algomerado de Virgem, e essa justificativa é corroborado por uma possível medida de anisotropia que pode indicar uma fonte extragaláctica próxima com um espectro de aceleração tão achatado quanto ao espectro detectado após o tornozelo.

O que pode-se retirar de informação destes três possíveis cenários é que somente é possível a origem de um raios cósmico ultra energético (UHECR - Ultra High Energy Cosmic Ray) se medirmos o espectro, anisotropia e a sua composição simultaneamente, e preferencialmente 1 a 1, assim como são os trabalhos atuais sobre a produção de neutrinos.

Para valores baixos na abscissa temos que o fluxo de raios cósmicos é grande a ponto de ser detectável diretamente, eis então que podemos nos perguntar, como detecta-se os raios cósmicos com baixo fluxo mas alta energia. Eis então que surge os grandes parques de detecção, onde são instalados vários detectores ao longo de uma grande área, mas por que ao longo de uma grande área? Para respondermos isso temos que voltar para os primórdios da física dos raios cósmicos, em 1938, Pierre
Auger prediz que ao adentrar a atmosfera a partícula irá interagir com os núcleos presentes formando um efeito de cascata.

Porém, os resultados mais animadores sobre esta classe de Raios Cósmicos vieram de experimentos de detecção de neutrinos [20]. A detecção de um neutrino ultraenergético permitiu a identificação de sua fonte, devido ao mecanismo discutido por [19], da interação com a radiação cósmica de fundo. Traçando a posição de origem desta partícula [21], verificou-se a existência de um blazar, um objeto da classe das AGNs. Por definição, é um corpo celeste que apresenta uma fonte de energia compacta e altamente variável, associada a um buraco negro supermassivo no centro de uma galáxia ativa. Portanto, a partir da detecção de neutrinos GZK podemos definir as fontes de raios cósmicos ultraenergéticos.

\section{Comentários finais}

Neste trabalho, apresentamos os cálculos sobre a componente eletromagnética dos raios cósmicos. Em especial, nos dedicamos a detalhar o mecanismo de interação de uma partícula com a radiação cósmica de fundo, que leva ao corte GZK [2,3], um limite para a energia de um raio cósmico, acessível a detectores localizados no solo da Terra. Para realizar tal cálculo, utilizamos conhecimentos de Eletromagnetismo e de Relatividade Especial, permitindo que mesmo o estudante ainda não familiarizado com a física de partículas, e com as interações fortes, possam acompanhar o texto aqui escrito.

Apesar de não poder considera o texto como introdutório, no sentido de estar disponível para alunos ingressantes no Ensino Superior, podemos classificá-lo como básico para quem gostaria de se aventurar nos estudos da física de partículas. Neste sentido, os mecanismos de interação aqui descritos e calculados são acessíveis aos alunos que já tiveram uma apresentação formal ao Eletromagnetismo, podendo ser trabalhado a partir do final do $3^{\mathrm{o}}$ semestre de Física Básica.

Nosso intuito, a partir da escrita deste trabalho, é proporcionar tal material de consulta, e permitir que mais estudantes de Física possam interessar-se pelos aspectos de pesquisa em altas energias. Aos já iniciados na área, podemos dizer que o mecanismo de aceleração para os raios cósmicos, outro ponto a ser estudado, não foi incluído neste texto para evitar que o mesmo se tornasse muito longo. Outro trabalho, apresentando os mecanismos de aceleração, está em preparação pelos autores.

\section{Referências}

[1] F.K. Nóbrega e L.F. Mackedanz, Rev. Bras. Ens. Fís. 35, 1 (2013).

[2] K. Greisen, Physical Review Letters 16, 748 (1966).

[3] G.T. Zatsepin e A.K. Vadem, Journal of Experimental and Theoretical Physics Letters 4, 78 (1966). 
[4] F.K. Nóbrega e L.F. Mackedanz, Rev. Bras. Ens. Fís. 40, e3318 (2018)

[5] D. Pacini, arXiv:1002.1810 (2010).

[6] D. Pacini, Il Nuovo Cimento 3, 93 (1912).

[7] V.F. Hess, Physikalische Zeitschrift 13, 1084 (1912).

[8] M.C. Bustamante, Rev. Bras. Ens. Fís. 35, 2603 (2013).

[9] P. Carlson e A. Angelis, Eur. Phys. J. H 35, 309 (2010).

[10] K. Kurz, Physikalische Zeitschrift 10, 834 (1909).

[11] A. Angelis, P. Carlson, N. Giglietto e S. Stramaglia, 32nd International Cosmic Rays Conference Proceedings (IHEP, Beijing, 2011).

[12] A.A. Pacini, Rev. Bras. Ens. Fís. 39, e1306 (2017).

[13] W. Bothe e W. Kolhörster, Naturwissenschaften 17, 271 (1929).

[14] A. Letessier-Selvon e T. Stanev, Reviews of modern physics 83, 907 (2011).

[15] T. Stanev, High energy cosmic rays (Springer Science \& Business Media, Berlin, 2010).

[16] P. Sokolsky Introduction to Ultrahigh Energy Cosmic Rays Physics (Westview Press, Colorado, 2004).

[17] A.G. Oliveira, M. Rockenbach e A.A. Pacini, Rev. Bras. Ens. Fís. 36, 1 (2014).

[18] K. Mannheim e R. Schlickeiser, Astronomy and Astrophysics 286, 983 (1994).

[19] F. Halzen e J. Phys, Conf. Ser. 337, 012050 (2012).

[20] Icecube Collaboration, Science 361, 147 (2018).

[21] https://science.sciencemag.org/content/361/6398/eaat1378/tab-pdf. 